\title{
Luminosity limit due to the beam-beam interactions with or without crossing angle
}

\author{
K. Ohmi, ${ }^{1}$ M. Tawada, ${ }^{1}$ Y. Cai, ${ }^{2}$ S. Kamada, ${ }^{1}$ K. Oide, ${ }^{1}$ and J. Qiang ${ }^{3}$ \\ ${ }^{1}$ KEK, 1-1 Oho, Tsukuba, 305-0801, Japan \\ ${ }^{2}$ Stanford Linear Accelerator Center, Menlo Park, California 94025, USA \\ ${ }^{3}$ Lawrence Berkeley National Laboratory, Berkeley, California 94720, USA
}

(Received 10 May 2004; published 14 October 2004)

\begin{abstract}
In recent high luminosity colliders, the finite crossing angle scheme becomes popular to gain the luminosity with multibunch or long bunch operation. Success of the KEKB factory showed that the finite crossing angle scheme has no problem achieving beam-beam parameters up to 0.05 . We have studied the beam-beam interactions with and without crossing angle toward higher luminosity. We discuss how the crossing angle affects the beam-beam parameter and luminosity in the present KEKB using computer simulations. The simulations showed that crab cavities, which realize the head-on collision effectively, can be expected to double the luminosity.
\end{abstract}

DOI: 10.1103/PhysRevSTAB.7.104401

PACS numbers: 29.20.Dh, 29.27.-a, 41.75.Ht

\section{INTRODUCTION}

High-luminosity B factories, KEKB and PEP-II, are operated successfully at KEK and SLAC, respectively. The collision scheme in KEKB was designed so that the two beams collide with a finite crossing angle, $2 \times$ $11 \mathrm{mrad}$, with $2 \mathrm{~ns}$ repetition. A head-on collision scheme was adopted in PEP-II with 4 ns repetition. The crossing angle makes it easy to design the interaction region for narrow bunch spacing. Historically, the crossing collision scheme had been considered to be taboo due to the unsuccessful experience of DORIS in DESY where a vertical crossing was implemented. At KEK, many studies were performed leading to the adoption of the horizontal crossing collision scheme [1-3]. KEKB and PEP-II achieved luminosities of $1.2 \times 10^{34}$ and $0.8 \times 10^{34} \mathrm{~cm}^{-2} \mathrm{~s}^{-1}$, respectively, in the beginning of 2004. Such high luminosities were not believed to be possible when the original design work started. But following the recent success of the high luminosity factories, the crossing angle scheme has become popular in order to gain a higher repetition rate.

The design luminosity at KEKB is achieved with the repetition frequency that is a factor of 3-4 lower than its design value. Currently, the bunch spacing, which is the inverse of the repetition frequency, is 8-6 ns. Narrowing the bunch spacing does not increase the luminosity, perhaps due to the electron cloud effect in both machines.

The bunch spacing has reached its design value, $4 \mathrm{~ns}$ for PEP-II, but the bunch pattern still consists of many small gaps between the bunch trains to alleviate the electron cloud effects. The number of bunches is much more than that of KEKB. The influence due to the electron cloud in PEP-II seems to be less a problem than that in KEKB. This fact may mean that the antechamber which is used in PEP-II is efficient in reducing the electron cloud effects.

The bunch population is much higher than its design to recover the smaller number of bunches in KEKB: it is twice the design for the positron beam and is 3.7 times the design for the electron beam. The operating tune, which optimizes the luminosity [4], is just slightly above a halfinteger in the horizontal and is around $0.54-0.56$ in the vertical. The horizontal beam-beam parameter, calculated using the bunch population and the design beam size, is about 0.1 , while the vertical beam-beam parameter, estimated from the luminosity, is 0.05 . Our design beam-beam parameter is 0.05 in both the horizontal and the vertical plane for the design bunch populations, which is much smaller than the present values. The vertical beam-beam parameter, which is reduced by a vertical beam enlargement, determines the luminosity performance.

We continue to make efforts to obtain higher luminosity. The achieved beam-beam parameter, 0.05 , is not the largest achieved value yet. For example, the beam-beam parameter, $\sim 0.07$, was reached in CESR with a similar damping time $\tau_{x y} / T_{0}$. To reach luminosity much higher than $10^{35} \mathrm{~cm}^{-2} \mathrm{~s}^{-1}$, a beam-beam parameter beyond 0.1 is necessary.

To study the effect of crossing angle, the beam-beam simulation has to take into account the bunch length; that is, the motion of particles should be treated in threedimensional space $(x-y-z)$. Both weak-strong and strong-strong simulations are used for the studies in this paper, where both of the two colliding beams are represented by a number of macroparticles in strong-strong simulations, while one (weak) beam is represented by a number of macroparticles and another (strong) beam is represented by a rigid charge distribution in weak-strong simulations. Weak-strong simulations have been extended to three-dimensional space by slicing the bunch along the longitudinal direction using the so-called synchrobeta map [5-7]. Most of the strong-strong simulations had been done in two-dimensional space $(x-y)$ [8]; i.e., the bunch length was assumed to be zero. Though strongstrong simulations have been extended to three- 
dimensional space, the convergence for the number of the longitudinal slices was not good $[9,10]$. The convergence has been improved dramatically by using an idea similar to the synchrobeta map as done in the weak-strong simulation [11]. This three-dimensional formalism is discussed in this paper.

The beam-beam limit for collision with or without crossing angle is studied using this simulation method. A luminosity optimization for the optics parameter at the collision point has been done in KEKB [12]. Simulations have also shown that tuning for $x-y$ coupling and dispersion functions at the collision point affects the beambeam performance [4]. It is important to remove the errors of coupling and dispersion for luminosity optimization. As we show later, the crossing angle is considered as a kind of dispersion function at the collision point; therefore, it makes the beam-beam performance worse.

This paper consists of the following contents. The formalism used in our simulation is discussed in Sec. II, which includes an explanation of the treatment of crossing angle and three-dimensional beam-beam interaction. Results obtained by the simulation are presented in Sec. III, and conclusions are summarized in Sec. IV.

\section{FORMALISM OF COLLISION WITH CROSSING ANGLE}

\section{A. Treatment of the crossing angle}

In the laboratory frame, the $s$ axes of two beams are chosen to be their moving directions, and the electromagnetic field of a beam is formed on the plane perpendicular to its $s$ axis. When there is a crossing angle in the collision, the beam-beam force by the other beam is not in the transverse direction of the beam that is experiencing the force. Hence the timing, when the beam experiences the force, depends on the positions of both beams. This feature makes it more difficult to treat the collision with a finite crossing angle.

It is convenient that the collision is treated by a Lorentz boost to a head-on frame from the laboratory frame $[1,2,13]$. In the head-on frame, the $s$ axes of the two beams coincide with opposite directions. The particles in the two beams basically move along the $s$ direction and beam-beam force is perpendicular to the $s$ axis. In the simulation, particles in the beam are transferred to the head-on frame, experience the collision, and are transferred back to the laboratory frame by the inverse of the Lorentz boost. The principle axis of the beam ellipsoid does not coincide with the $s$ direction; that is, $\langle x z\rangle$ is not zero for the case of horizontal crossing.

We use as dynamical variable $\boldsymbol{x}(s)=$ $\left(x, p_{x}, y, p_{y}, z, p_{z}\right)^{t}$, which is generally used to describe beam motion in accelerators: $p_{x, y}$ is the transverse momentum normalized by the total momentum of a reference particle $\left(p_{0}=E_{0} / c\right), z$ is the delay time $(z=$ $s-c t)$ scaled by the light speed, and $p_{z}$ is the energy deviation $\left[p_{z}=\left(E-E_{0}\right) / E_{0}\right]$ from that of the reference particle.

The Lorentz transformation from the laboratory frame to the head-on frame $\left(\mathcal{M}_{L}\right)$ is given for a half-crossing angle $\theta$ by [2]

$$
\begin{aligned}
& x^{*}=\tan \theta z+\left(1+\frac{p_{x}^{*}}{p_{s}^{*}} \sin \theta\right) x, \quad y^{*}=y+\sin \theta \frac{p_{y}^{*}}{p_{s}^{*}} x, \\
& z^{*}=\frac{z}{\cos \theta}-\frac{H^{*}}{p_{s}^{*}} \sin \theta x, \quad p_{x}^{*}=\frac{p_{x}-\tan \theta H}{\cos \theta} \\
& p_{y}^{*}=\frac{p_{y}}{\cos \theta}, \quad p_{z}^{*}=p_{z}-\tan \theta p_{x}+\tan ^{2} \theta H
\end{aligned}
$$

where

$$
\begin{aligned}
& H=\left(1+p_{z}\right)-\sqrt{\left(1+p_{z}\right)^{2}-p_{x}^{2}-p_{y}^{2}}, \\
& p_{s}=\sqrt{\left(1+p_{z}\right)^{2}-p_{x}^{2}-p_{y}^{2}} .
\end{aligned}
$$

A star designates a dynamical variable in the head-on frame. $H^{*}$ and $p_{s}^{*}$ are $H\left(\boldsymbol{p}^{*}\right)$ and $p_{s}\left(\boldsymbol{p}^{*}\right)$, respectively. Note that the $x^{*}$ and $y^{*}$ axes are defined in the same direction for both beams, while the $s^{*}$ axis is defined in opposite directions, since the two beams travel in opposite directions.

The linear part of the transformation is expressed by a matrix

$$
M_{L}=\left(\begin{array}{cccccc}
1 & 0 & 0 & 0 & \tan \theta & 0 \\
0 & 1 / \cos \theta & 0 & 0 & 0 & 0 \\
0 & 0 & 1 & 0 & 0 & 0 \\
0 & 0 & 0 & 1 / \cos \theta & 0 & 0 \\
0 & 0 & 0 & 0 & 1 / \cos \theta & 0 \\
0 & -\tan \theta & 0 & 0 & 0 & 1
\end{array}\right)
$$

These transformations, Eqs. (1) and (2), are not symplectic. In fact, the determinant of the transfer matrix $M_{L}$ is not 1 , but $\cos ^{-3} \theta$. This is due to the fact that the Lorentz transformation is not symplectic for the accelerator coordinate, because the Hamiltonian is divided by a reference momentum. This is not a problem because the inverse factor of $\cos ^{3} \theta$ is applied by the inverse transformation.

This discussion can be applied to the nonlinear transformation of Eq. (1). The Jacobian matrix of the transformation, $\left(\partial \boldsymbol{x}^{*} / \partial \boldsymbol{x}\right)_{i j}=\partial x_{i}^{*} / \partial x_{j}$, satisfies

$$
\left(\frac{\partial x^{*}}{\partial x}\right)^{t} J\left(\frac{\partial x^{*}}{\partial x}\right)=J / \cos \theta
$$

where $J$ is the matrix of the symplectic metric. Note that $\operatorname{det}(J / \cos \theta)=\cos ^{-6} \theta . M^{t}$ denotes the transpose of the matrix $M$. Once again, it shows that the Lorentz transformation itself is not symplectic but the whole beambeam map that includes the Lorentz transformation, beam-beam map, and the inverse Lorentz transformation is symplectic, since 


$$
X^{t} J X=J
$$

where $X$ is the product of three Jacobian matrices:

$$
X=\left(\frac{\partial \boldsymbol{x}}{\partial \boldsymbol{x}^{*}}\right)_{L^{-1}}\left(\frac{\partial \boldsymbol{x}^{*}}{\partial \boldsymbol{x}^{*}}\right)_{\text {beam-beam }}\left(\frac{\partial \boldsymbol{x}^{*}}{\partial \boldsymbol{x}}\right)_{L} .
$$

The map of the beam-beam interaction, which includes the collision along the bunch length, is represented by a map at $s^{*}$ as shown in the next subsection. The revolution map including the crossing transformation, is expressed by

$$
\mathcal{M}_{L^{-1}} \circ \mathcal{M}_{\text {arc }} \circ \mathcal{M}_{L}
$$

where $\mathcal{M}_{\text {arc }}$ is the transfer map of the arc section and $\mathcal{M}_{L}$ is the map given by Eq. (1). The linear part of the revolution map, which is the revolution matrix, is expressed by $M_{L} M_{\text {arc }} M_{L}^{-1}$. For small $\theta$, the revolution matrix, including the crossing transformation, is equivalent to that with $z$-dependent dispersion $\zeta_{x}=\theta$ [14] at the interaction point, $s=s^{*}$. The $z$-dependent dispersion is an orbit displacement as a linear coefficient for $z$, i.e., $\zeta_{x}=\Delta x / z$, while the ordinary dispersion is an orbit displacement for $p_{z}, \eta_{x}=\Delta x / p_{z}$. The equilibrium beam envelope $\left(\left\langle x_{i}^{*} x_{j}^{*}\right\rangle\right)$ at the collision point is determined by the optics function [14]. The effective $\zeta$ at the collision point means that the beam envelope has $\langle x z\rangle \propto$ $\zeta_{x}(s)$ in the head-on frame. The nonlinear parts of the transformations of Eq. (1) are a kind of kinematic term which only weakly affect the beam for a small crossing angle.

Since crab cavities also create a $z$-dependent dispersion $\zeta_{x, \text { crab }}(s)$, we can effectively choose an arbitrary crossing angle by using the crab cavities to control $\zeta_{x, \text { crab }}\left(s^{*}\right)$ at the interaction point. To make a head-on collision of the beams with $\langle x z\rangle=0$, we need to set $\zeta_{x \text {, rrab }}=-\theta$.

We use two simulation models, weak-strong and strong-strong, to study the beam-beam interactions according to circumstance. In the weak-strong model, the strong beam is fixed to a Gaussian distribution in sixdimensional phase space, while the other (weak) beam is represented by macroparticles. We restrict ourselves to these two methods to avoid complexity and confusion. We have two other simulation methods, which are not treated in this paper. They are strong-strong simulation with a Gaussian approximation and weak-strong simulation with the particle-in-cell (PIC) method $[15,16]$. In the first method, both beams, which are represented by macroparticles, are approximated by Gaussian distributions turn by turn. In the second method, the strong beam, which has a given arbitrary charge distribution, drives macroparticles in the weak beam with a force determined by the PIC method.

In the weak-strong model, macroparticles are transferred from the laboratory frame to the head-on one using Eq. (1). The beam envelope of the strong beam is trans- ferred by the linear map,

$$
\left\langle\boldsymbol{x}^{*} \boldsymbol{x}^{* t}\right\rangle=M_{L}\left\langle\boldsymbol{x} \boldsymbol{x}^{t}\right\rangle M_{L}^{t}
$$

In the strong-strong model, both of the beams are represented by macroparticles, which can have an arbitrary distribution. The beam-beam force is calculated by the PIC method [17]. Macroparticles of both beams are transferred by Eq. (1).

\section{B. Formalism of three-dimensional beam-beam in- teraction}

We now consider the collision of two beams in the head-on frame. The beam-beam force is perpendicular to the $s$ axis due to the Lorentz transformation to the head-on frame. Therefore it is sufficient to take care of the beam shapes deformed by the crossing transformation of Eq. (1). The two beams both have gradients in the $x-z$ plane due to the crossing angle. It is essential to take into account the bunch length, since the effect of the gradient in the $x-z$ plane should be studied. For simplicity, the collision point is chosen as $s^{*}=0$. The transfer map before $(s=-0)$ to after $(s=+0)$ the collision in the head-on frame, evaluated at the interaction point $s=$ $s^{*}=0$, is written as

$$
\boldsymbol{x}(+0)=S \exp \left[-: \int_{-\Delta}^{\Delta} V_{0}^{-1}(s) H_{b b} V_{0}(s) d s:\right] \boldsymbol{x}(-0),
$$

where $S$ means $s$ ordered product and $\pm \Delta$ is the interaction region of the two beams. ${ }^{1} V_{0}$ is the transfer map in the drift space,

$$
\begin{aligned}
V_{0}(s) & \equiv V_{0}(s, 0)=S \exp \left[-: \int_{0}^{s} H_{0} d s:\right] \\
& =\prod_{i= \pm} \exp \left[-: \frac{p_{x, i}^{2}+p_{y, i}^{2}}{2} s:\right]
\end{aligned}
$$

where $p_{x(y), \pm}$ is the momentum of positrons/electrons. Note that $: p_{x, \pm}: x_{ \pm}=\left[p_{x, \pm}, x_{ \pm}\right]=-1$ and $: p_{x, \pm}: x_{\overline{+}}=0$, where [] is the Poisson bracket. The drift map $V_{0}$ can be replaced by the map in the solenoid magnet as the need arises. $H_{b b}$ is a term which represents the beam-beam interaction. The relativistic beam induces an electromagnetic field in the transverse plane. The field can be expressed by a two-dimensional static potential. The other beam experiences the electromagnetic field. $H_{b b}$ is expressed by

$$
H_{b b}=\phi_{-}\left(\boldsymbol{x}_{+}\right)+\phi_{+}\left(\boldsymbol{x}_{-}\right),
$$

where $\phi_{\mp}\left(\boldsymbol{x}_{ \pm}\right)$is the potential induced by $e^{\mp}$ beam at the position $\left(\boldsymbol{x}_{ \pm}\right)$of each $e^{ \pm}$in their beam.

\footnotetext{
${ }^{1} S$ ordered product is the same concept as $T$ ordered product popularly used.
} 
We have two problems when evaluating the map, Eq. (8): that is, one is how to evaluate $\phi$, and the second is how to integrate Eq. (8) for a given $\phi$. The potential $\phi$ is determined by the two-dimensional Poisson equation,

$$
\triangle_{\perp} \phi_{ \pm}(x, y ; z, s)=\frac{r_{e}}{\gamma_{ \pm}} \rho_{ \pm}(x, y ; z, s),
$$

where $\rho$ is the number density of the beam at given $(z, s)$. $(x, y, z, s)$ are coordinates of each $e^{\mp}$ in their beam, which is tracked. The potential is solved by the PIC method.

We discuss the motion of the positron beam which experiences the potential formed by the electron beam, for example. The integration including an $S$ ordered product is discretized. It is convenient that the integration variable $(s)$ is replaced by $z_{-}$for the beam of the field source, and the integration is discretized for $z_{-}$of the source beam, $z_{-, i}$. The integration step is chosen to be the slice in which the potential is calculated. The relation between $s_{i}$ and $z_{-, i}$ is expressed by $s_{i}=\left(z_{+}-z_{-, i}\right) / 2$. The integration for both electron and positron beams is expressed by

$$
\begin{aligned}
& \prod_{i=1}^{N_{s l,-}} \exp \left[-: V_{0,+}^{-1}\left(s_{-, i}\right) \phi_{-, i}\left(\boldsymbol{x}_{+}, s_{-, i}\right) V_{0,+}\left(s_{-, i}\right) \Delta s:\right] \\
& \quad \times \prod_{j=1}^{N_{s l,+}} \exp \left[-: V_{0,-}^{-1}\left(s_{+, j}\right) \phi_{+, j}\left(\boldsymbol{x}_{-}, s_{+, j}\right) V_{0,-}\left(s_{+, j}\right) \Delta s:\right]
\end{aligned}
$$

Since $\phi_{ \pm, i}(x)$ is determined by the distribution of the $e^{ \pm}$beam at $s_{\bar{\mp}, i}$, then $s_{\bar{\mp}, i}$ is a function of the longitudinal position of the tracked particle in the beam, $z_{ \pm}$. Namely, the potential has to be calculated for every tracked particle.

For a Gaussian beam, it is easy to get $\phi$ as a function of $s[7,18]$,

$$
\begin{aligned}
\phi_{ \pm}(x, y, z ; s)= & \frac{N_{ \pm}}{4 \pi \sigma_{x} \sigma_{y}} \int d x^{\prime} d y^{\prime} \ln \mid\left(x-x^{\prime}\right)^{2}+(y \\
& \left.-y^{\prime}\right)^{2} \mid \exp -\left(\frac{x^{\prime 2}}{2 \sigma_{x}(s)^{2}}+\frac{y^{\prime 2}}{2 \sigma_{y}(s)^{2}}\right) .
\end{aligned}
$$

Its derivative is expressed by the complex error function [19]. The beam-envelope matrix is deformed due to the beam-beam interaction at each integration step. For an arbitrary $s$ in an integration step, the rms beam sizes, or more generally beam-envelope matrix, are transferred by

$$
\left\langle\boldsymbol{x}\left(s^{\prime}\right) \boldsymbol{x}^{t}\left(s^{\prime}\right)\right\rangle=V_{0}\left(s^{\prime}, s\right)\left\langle\boldsymbol{x}(s) \boldsymbol{x}^{t}(s)\right\rangle V_{0}^{t}\left(s^{\prime}, s\right),
$$

where $V_{0}\left(s^{\prime}, s\right)$ is the transfer matrix for $s$ to $s^{\prime}$.

In the weak-strong simulation, a strong beam is fixed in charge distribution in every longitudinal integration step. The beam-envelope matrix is transferred by Eq. (14) during the collision $[6,7]$.
In the PIC model, the distribution is expressed by macroparticles mapped onto a two-dimensional grid space; therefore it is difficult to express the potential as a function of $s$. The calculation of the potential in every $s$ for $z$ of a tracked particle is impossible because of the enormous computing power required. Therefore we use an interpolation of the potential during the integration step. The two-dimensional potential, which determines the beam-beam force, is estimated at a collision point front $\left[s_{f}=\left(z_{i, f}-z_{j}\right) / 2\right]$ and back $\left[s_{b}=\left(z_{i, b}-z_{j}\right) / 2\right]$ face of the (ith) slice containing the particle. The potential of the target slice $\phi(s)$ is transferred to the collision point of the particle by a linear interpolation,

$$
\phi_{j}(s)=\phi_{j}\left(s_{b}\right)+\frac{\phi_{j}\left(s_{f}\right)-\phi_{j}\left(s_{b}\right)}{s_{f}-s_{b}}\left(s-s_{b}\right)
$$

where $s$ depends on $z$ as $s=\left(z-z_{j}\right) / 2$. An energy change proportional to $\partial \phi / \partial z$, which is caused by the interaction depending on $z$, is included in the simulation,

$$
\Delta p_{z}=-\frac{\phi_{j}\left(s_{f}\left(z_{i, f}\right)\right)-\phi_{j}\left(s_{b}\left(z_{i, b}\right)\right)}{z_{i, f}-z_{i, b}} .
$$

The number of slices required depends on the beambeam parameter. The distribution of the beam is deformed during the process of the collision. The disruption parameter is mainly important in the vertical. It is an oscillation angle of a particle in the potential of the other beam and characterizes the deformation [18,20,21]

$$
\chi=\sqrt{\frac{4 \pi \bar{\xi}_{y} \sigma_{z}}{\beta_{y}}} .
$$

The oscillation angle is divided by the integration step along the longitudinal slice. The angle in each step should be smaller than 1; i.e., a high beam-beam disruption requires a large number of slices. The disruption parameter is $\chi \approx 1.1-1.6$ for our parameter region, $\xi_{y}=0.1-0.2$ and $\sigma_{z} \approx \beta_{y}$. The simulation results converged for the slice number of 5-10; i.e., the divided angle is $0.1-0.3$ for each slice. A larger number is better, but the calculation time grows as the square of the slice number. Figure 1 shows the evolution of the beam-beam parameter for 5 , 10, and 15 slices. The beam-beam parameter converges completely for ten slices. There is a difference, less than $5 \%$, in the final beam-beam parameters between five and ten slices. In this paper, simulation results are given for five slices. Figure 2 shows variation of beam potential along $s$ for $N_{s l}=5$. The potential is given for particles colliding with the third (center) slice. Figures 2(a) and 2(b) depict $\phi_{3}(s)$ and $k_{y}=$ $\partial^{2} \phi(3) / \partial y^{2}=\Delta p_{y} / \Delta x$. Note that collision starts from particles with larger $s$. Gaps, which are seen between steps in the pictures, arise from disruption due to interaction with previous slices. 


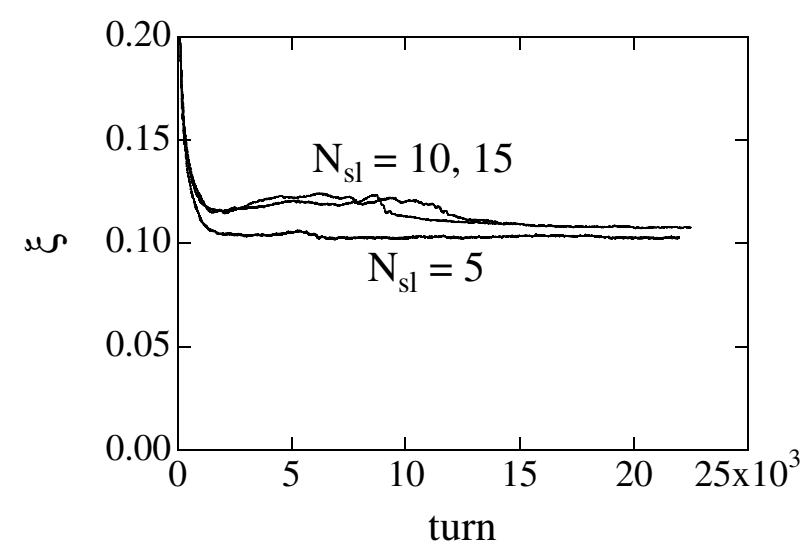

FIG. 1. Evolution of the beam-beam parameter for $N_{s l}=5$, 10 , and 15 .

\section{SIMULATION RESULTS}

The effects of crossing angle on the beam-beam parameter and luminosity are studied for the machine parameters as is shown in Table I. The parameters in the
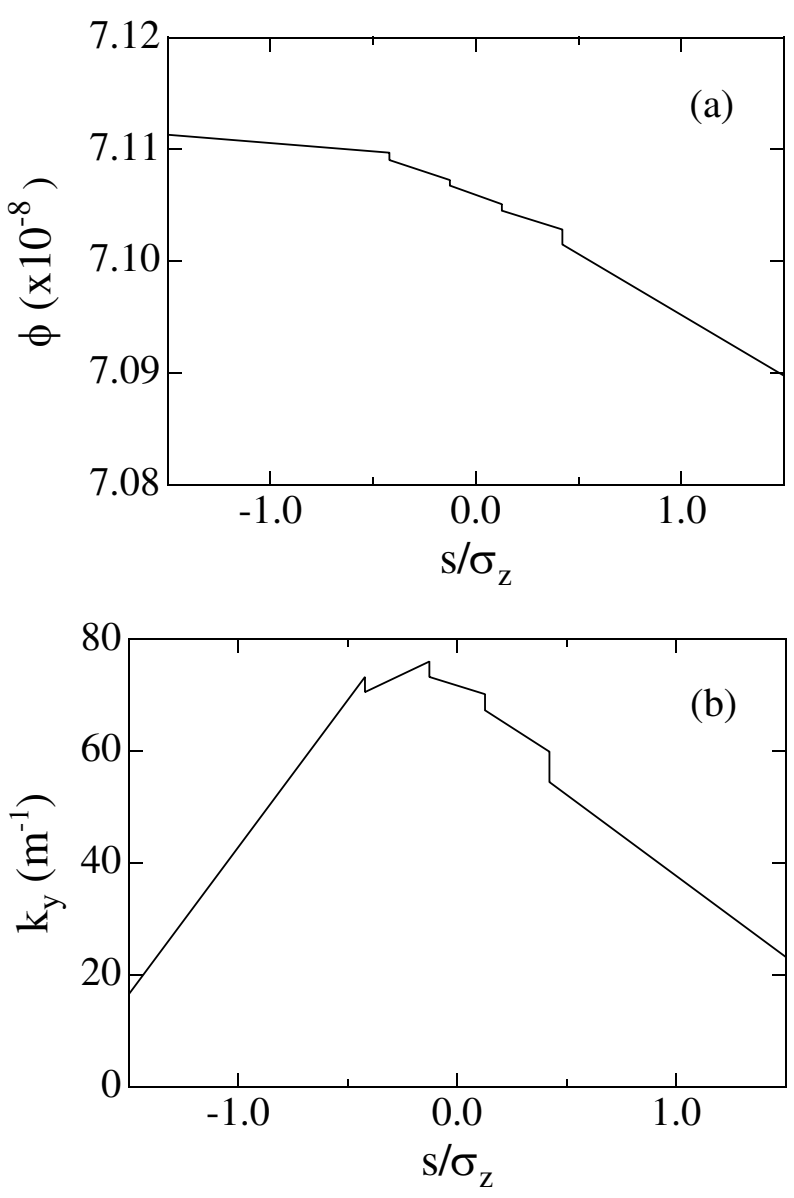

FIG. 2. Variation of beam potential along $z$ for $N_{s l}=5$. The potential is given for particles colliding with the third (center) slice. (a),(b) depict $\phi_{3}(s)$ and $k_{y}=\partial^{2} \phi(3) / \partial y^{2}=\Delta p_{y} / \Delta y$. Note that collision starts from particles with larger $s$.
TABLE I. Basic parameters of KEKB.

\begin{tabular}{|c|c|c|}
\hline & HER & LER \\
\hline$C$ & \multicolumn{2}{|c|}{$3016 \mathrm{~m}$} \\
\hline E & $8 \mathrm{GeV}$ & $3.5 \mathrm{GeV}$ \\
\hline$\beta_{x} / \beta_{y}$ & \multicolumn{2}{|c|}{$60 \mathrm{~cm} / 7 \mathrm{~mm}$} \\
\hline$\varepsilon_{x} / \varepsilon_{y}$ & \multicolumn{2}{|c|}{$18 \mathrm{~nm} / 0.18 \mathrm{~nm}$} \\
\hline$\sigma_{z}$ & \multicolumn{2}{|c|}{$7.0 \mathrm{~mm}$} \\
\hline$\nu_{x} / \nu_{y}$ & \multicolumn{2}{|c|}{$0.515 / 0.58 / 0.016-0.024$} \\
\hline$\nu_{s}$ & \multicolumn{2}{|c|}{$0.016-0.024$} \\
\hline$\tau_{x y} / T_{0}$ & 4000 turn & 4000 turn \\
\hline$\theta_{c}$ & \multicolumn{2}{|c|}{$0-2 \times 11 \mathrm{mrad}$} \\
\hline
\end{tabular}

table are the same as those of the present KEKB except the transparency condition was kept as $N_{+} \gamma_{+}=N_{-} \gamma_{-}$to avoid complex behavior caused by unbalance of the beams in the simulation. The betatron tunes are equal to the operating point of the high energy ring (HER) of KEKB. By the way, the operating point of the low energy ring (LER) is slightly lower in both planes, namely, $(0.508,0.55)$.

In the weak-strong simulation, 100 macroparticles are tracked up to 40000 turns (10 times of the damping time; i.e., $10 \times \tau_{x(y)} / T_{0}$ ), and the luminosity was calculated by averaging it during 20000 through 40000 turns. In the strong-strong simulation, 100000 macroparticles are tracked up to 20000 turns. The transverse plane is divided into $128 \times 256$ grids with unit size of $20 \mu \mathrm{m} \times$ $0.4 \mu \mathrm{m}$. The luminosity was calculated using the beam particle distributions for the last turn. The bunch population of positrons $\left(N_{+}\right)$in the LER is scanned from $1 \times$ $10^{10}$ to $1.2 \times 10^{11}$. The beam-beam parameter was calculated using the luminosity as follows:

$$
\xi_{y}=\frac{2 r_{e} \beta_{y}}{N_{ \pm} \gamma_{ \pm}} \frac{L}{f_{\text {rep }}} .
$$

The beam-beam parameter is equal to the vertical incoherent beam-beam tune shift for collision between Gaussian beams, if the beam aspect ratio $\sigma_{y} / \sigma_{x}$ is approximated to be 0 and reduction factors due to the hourglass effect and crossing angle are neglected.

\section{A. Beam-beam limit for collisions with or without crossing angle}

The beam-beam limit phenomenon, in which the beam-beam parameter is saturated at a certain value as the current increases, is discussed first. The simulations were carried out for various bunch populations to obtain the limit of the beam-beam parameter. Figure 3 shows the beam-beam parameter $\xi$ as a function of the bunch population for collision with and without crossing angle. For the head-on collision, as seen in the figure, there is a significant difference between the beam-beam limits obtained by the weak-strong and the strong-strong simula- 

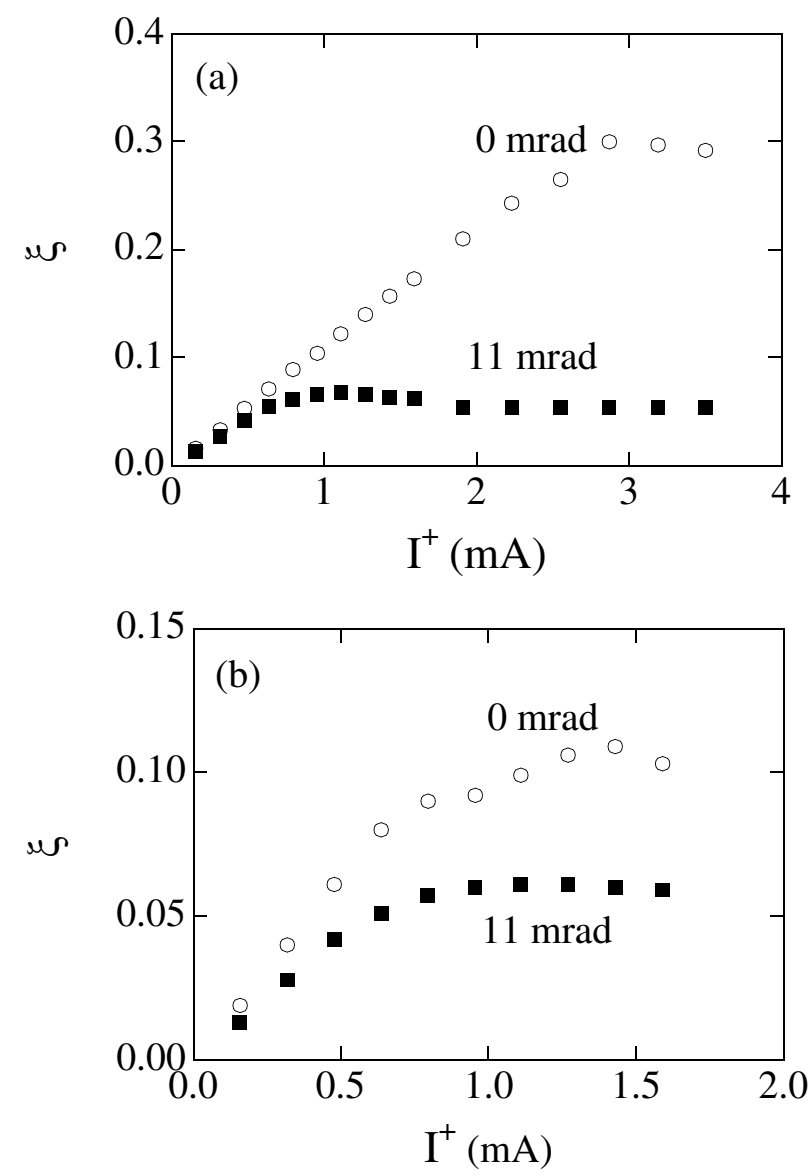

FIG. 3. Beam-beam parameters for crossing angle of 0 and $11 \mathrm{mrad}$ as function of bunch current. (a),(b) were obtained by the weak-strong and strong-strong simulations, respectively.

tions. The strong beam is assumed to be a Gaussian distribution in the weak-strong simulation, while arbitrary distributions are treated in the strong-strong simulation. The difference due to a non-Gaussian distribution is essential to determine the beam-beam limit [15]; that is, the accurate beam-beam limit is around $\xi \sim 0.1-0.12$. A strong-strong simulation with Gaussian approximation showed results similar to this weak-strong simulation $(\xi>0.2)$. A weak-strong simulation based on the PIC method showed results similar to this strong-strong simulation $(\xi \approx 0.1)$, if the distorted beam distribution given by this strong-strong simulation is used as the strong beam. The simulations, in which a Gaussian distribution is assumed, should not be used to predict the beam-beam limit.

With the half-crossing angle at $11 \mathrm{mrad}$, the beambeam parameter has similar behavior for both simulations; that is, $\xi$ is saturated around 0.06 . The beam-beam limit is about half of that in the head-on collision, though the geometrical reduction due to the crossing angle is about $20 \%$. A considerable luminosity loss is caused by the dynamical effects of collision when we have a crossing angle. We notice that the characteristics of the beam- beam limit are somewhat different from that for a headon collision; namely, the Gaussian approximation seems to be able to reliably predict the beam-beam limit for a collision with a large crossing angle as seen in Fig. 3. This fact also suggests that the beam-beam limit for a collision with a large crossing angle is caused by another mechanism.

\section{B. Crossing angle dependence}

The simulations for various crossing angles were performed in order to study the effect of crossing angle. Figure 4 shows the beam-beam parameter and beam sizes as functions of the crossing angle for the bunch population of $N_{+}=8 \times 10^{10}\left(I_{+}=1.1 \mathrm{~mA}\right)$. The beam-beam parameters given by the weak-strong and strong-strong simulations are depicted in Fig. 4(a). We had the same behavior for $N_{+}=10 \times 10^{10}$. The geometrical luminosity, which is also plotted in the picture, has a weak dependence on the crossing angle. The simulated luminosity has a peak structure near zero-crossing angle. The peak structure of the strong-strong simulation is narrower than that of the weak-strong simulation. The behavior of the horizontal and vertical beam sizes is depicted in Figs. 4(b) and 4(c), respectively. The horizontal beam size increases monotonically for crossing angle in both simulations. The vertical beam size has different behavior: the strong-strong simulation shows a large enlargement for small crossing angle of a few mrad and a gradual decrease for increasing crossing angle. The weak-strong simulation shows a monotonic increase with the crossing angle. This behavior in the strong-strong simulation is seen for high beam-beam parameter. When the current is decreased to be $N_{+}=3 \times 10^{10}\left(\xi_{\text {nom }}=0.05\right)$, the peak structure near zero-crossing angle was weakened.

\section{Diffusion due to crossing angle}

We are considering the origin of the luminosity degradation due to the crossing angle to be nonlinear diffusion $[16,22]$. The crossing angle is regarded as a dispersion that couples to $z$ at the collision point contrasted with the ordinary dispersion that couples to $p_{z}$. The linear couplings at the collision point in the $x-z$ plane induce nonlinear diffusions. In the weak-strong simulation with Gaussian approximation, luminosity degradation was seen, though the strength was somewhat weak; therefore some essentials of the degradation seem already included in the weak-strong model. The diffusion caused by the nonlinear dynamics of a single particle is treated by the weak-strong model. Though there may be more complex diffusion in which the two beams are strongly coupled, it is interesting to understand how the study based on single particle dynamics is helpful for our first step.

Synchrotron radiation also causes diffusion which determines the natural emittance due to the balance of the radiation damping when beam-beam or intrabeam effects 

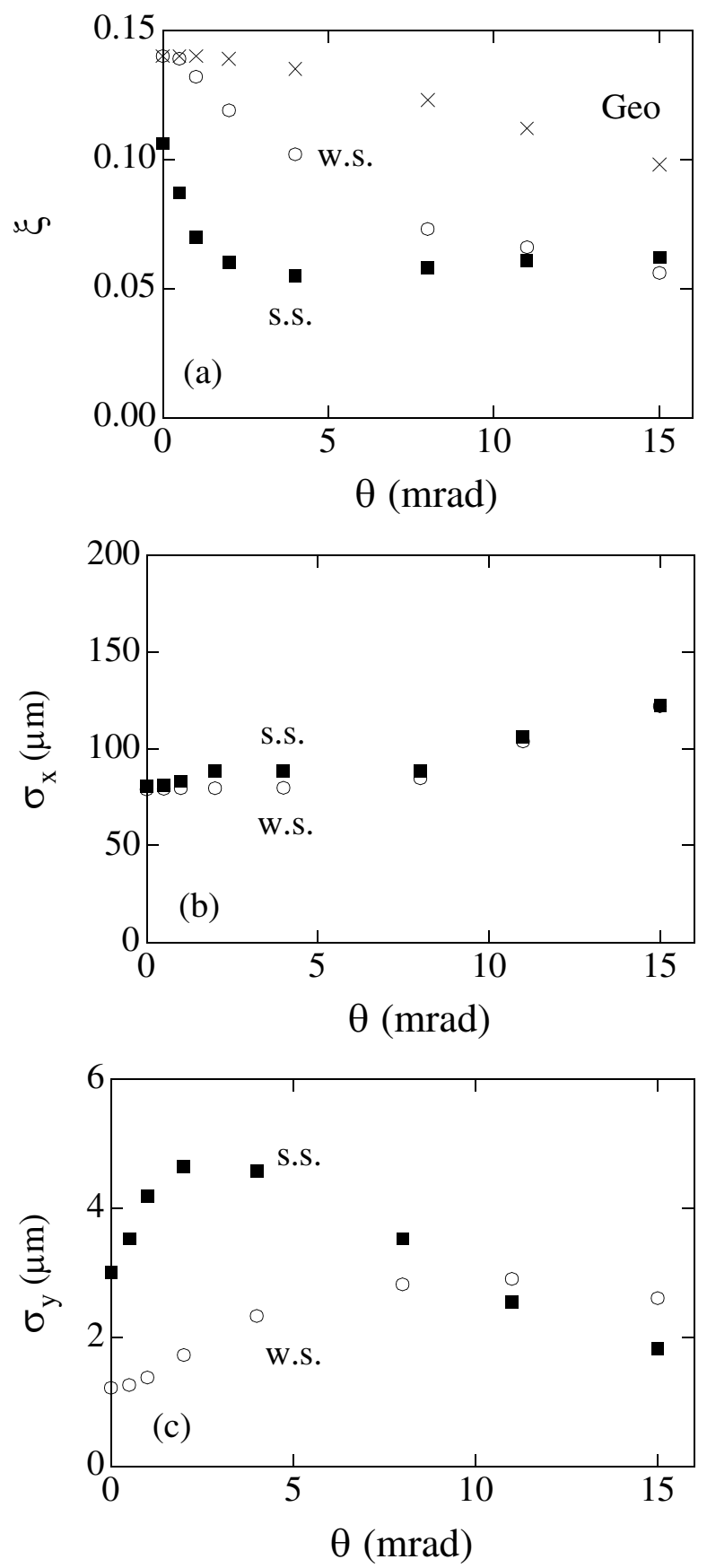

FIG. 4. Beam-beam parameters and beam sizes as a function of crossing angle. The beam-beam parameter, horizontal and vertical beam sizes, which are obtained by the strong-strong and weak-strong simulations, are depicted in (a)-(c), respectively.

are negligible. The beam size is determined by the ratio of the diffusion rate and damping rate,

$$
\sigma_{y}^{2}=\left\langle\Delta y^{2}\right\rangle \tau_{y} / 2 T_{0}
$$

where $\left\langle\Delta y^{2}\right\rangle$ and $T_{0} / \tau_{y}$ are the diffusion rate and the damping rate, respectively, per turn in the vertical. More sophisticated treatment is seen in Ref. [14]. The diffusions due to the synchrotron radiation are $\left\langle\Delta x_{\text {sr }}^{2}\right\rangle=$ $5.4 \mu \mathrm{m}^{2}$ and $\left\langle\Delta y_{\mathrm{sr}}^{2}\right\rangle=6.3 \times 10^{-4} \mu \mathrm{m}^{2}$. If two or more kinds of diffusion sources exist and are independent of each other, the total diffusion rate is the summation of individual diffusion rates. The diffusion due to the beambeam interaction is caused by the nonlinear force. There may be interferences among the diffusions if they are not independent.

To estimate the diffusion rate caused by the beambeam interaction, we remove the synchrotron radiation in the weak-strong simulations. The diffusion is observed in the growth of the beam size or, in other words, second order moments $\left(\left\langle x^{2}\right\rangle\right.$ and $\left.\left\langle y^{2}\right\rangle\right)$. Figures 5(a) and 5(b) show the evolution of the square of the horizontal and vertical beam sizes. They show a monotonic increase with respect to evolution turns. The diffusion rate is estimated by the growth rate per turn of the second moments $\left(\left\langle x^{2}\right\rangle\right.$ and $\left.\left\langle y^{2}\right\rangle\right)$. The figure shows that the crossing angle induces both horizontal and vertical diffusions, which rates are plotted in Fig. 5(c). The crossing angle, which causes linear $x-z$ coupling, causes not only the horizontal diffusion but also the vertical one. The horizontal diffusion is much smaller than that due to synchrotron radiation, but the vertical one is comparable with that due to synchrotron radiation. Figure 5(d) shows the vertical beam size estimated by the total diffusion of nonlinearity and radiation and the radiation damping time. The equilibrium beam size, obtained by the weak-strong simulation with synchrotron radiation, is also plotted in the figure. The beam size given by the weak-strong simulation is larger than that estimated by the diffusion rates.

It indicates that the total diffusion rate is larger than the summation of the two rates of radiation excitation and nonlinear diffusion. An interference between the radiation and nonlinear diffusions is discussed, for example, in Ref. [23]. Such an interference can occur for the case that a particle trajectory in the phase space (Poincaré plot) is stochastic. The particles come and go between large amplitudes even in a small amplitude change due to the radiation excitation. Clearly, the roles of the nonlinear diffusion in the beam-beam limit should be studied further at this point; this explanation should be considered as a conjecture.

We try to understand the behavior of the beam-beam limit as a function of crossing angle. The behavior of the weak-strong simulation is understood straightforwardly; that is, the strength of the diffusion reflects the beam size, though some interference between the two diffusions is seen quantitatively. A sharp luminosity drop with crossing angle, which is caused by a blowup of the vertical size, is observed in the strong-strong simulation. The beam-beam limit for head-on collision is reported to be caused by vertical distortion of the beam distribution from a Gaussian at this tune operating point [15]. The vertical enlargement was enhanced by the radiation ex- 

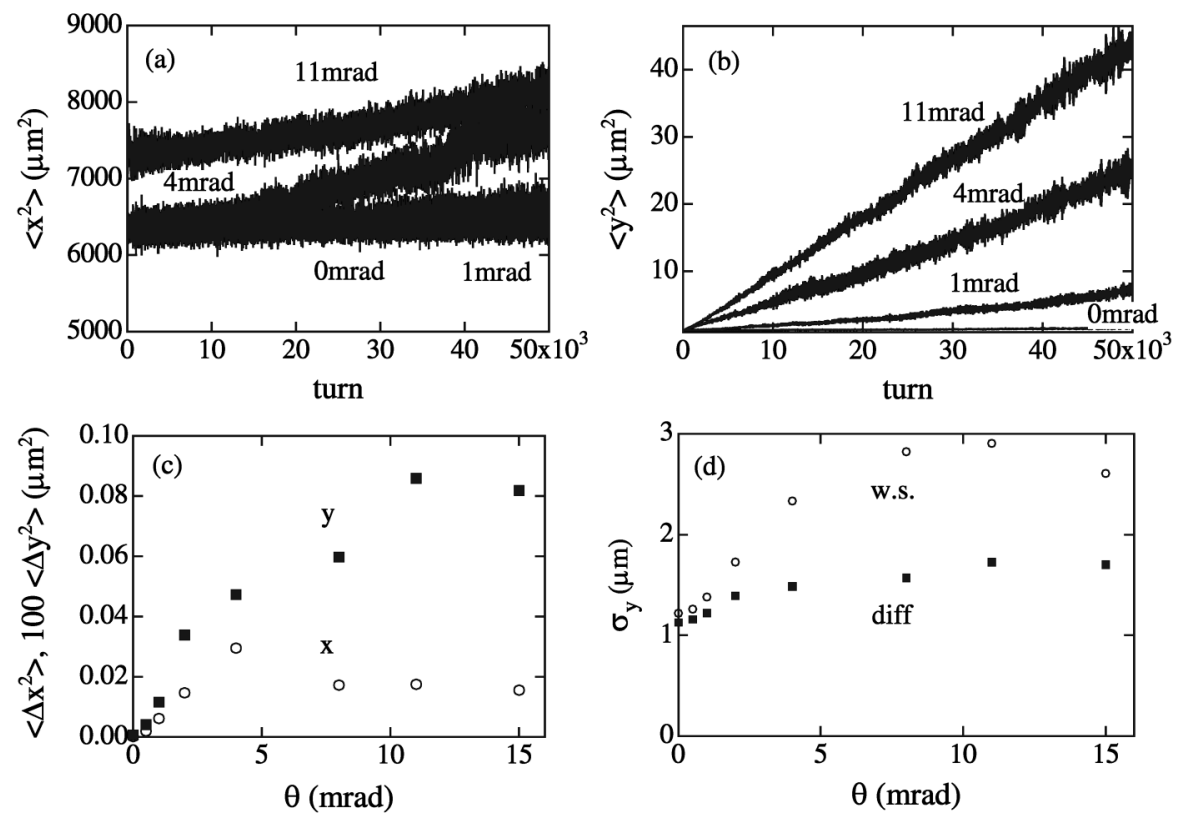

FIG. 5. Diffusion of horizontal and vertical beam size due to crossing angle. (a),(b) depict evolutions of horizontal and vertical beam sizes, respectively. (c) depicts the horizontal and vertical diffusion rates. (d) depicts vertical beam sizes, which are estimated by the diffusion rate (boxes) and are given by the weak-strong simulation with considering the synchrotron radiation (circles).

citation for head-on collision [16,22]. One possibility to explain the sharp structure in Fig. 4(a) is that the vertical enlargement is enhanced by the nonlinear diffusion.

\section{Tune survey}

We have discussed the beam-beam parameter at our design operating point shown in Table I. Simulations and experiments showed that this tune operating area is the best in the tune space; therefore our discussion has been limited to this operating point. It is well known that the operating tune strongly affects the beam-beam limit. We now investigate how the operating tunes affect the beambeam limit. The strong-strong simulation was done in the tune area $\left(0.508 \leq \nu_{x} \leq 0.548,0.51 \leq \nu_{y} \leq 0.63\right)$ with a step of 0.005 .

Figure 6 shows contour plots of the beam-beam parameter $\xi_{y}$ for collision with crossing angles of 0 and $11 \mathrm{mrad}$ in the tune area. The contour lines are drawn every 0.01 , and lighter gray corresponds to higher beambeam parameter. The best operating point is around $\left(\nu_{x}, \nu_{y}\right)=(0.508,0.55)$ for finite crossing angle, while it is around $(0.508,0.58-0.6)$ for zero-crossing angle. The best beam-beam parameters were 0.15 and 0.08 for a crossing angle of 0 and $11 \mathrm{mrad}$, respectively.

Non-Gaussian distribution was essential to determine the beam-beam limit for collision without crossing angle. Kurtosis, which is defined by $\left\langle y^{4}\right\rangle / 3\left\langle y^{2}\right\rangle^{2}$, is a measure of how the distribution is distorted away from Gaussian. Increase of kurtosis $k_{y} \sim 4$ was seen in the beam-beam limit [15], where $k_{y}=1$ for Gaussian distribution. Beam size and kurtosis, which were given by the tune survey, are shown in Figs. 7 and 8 for crossing angles of 0 and $11 \mathrm{mrad}$, respectively.

The behavior of the beam-beam parameter in Fig. 6(a), which is given for zero-crossing angle, is reflected in the
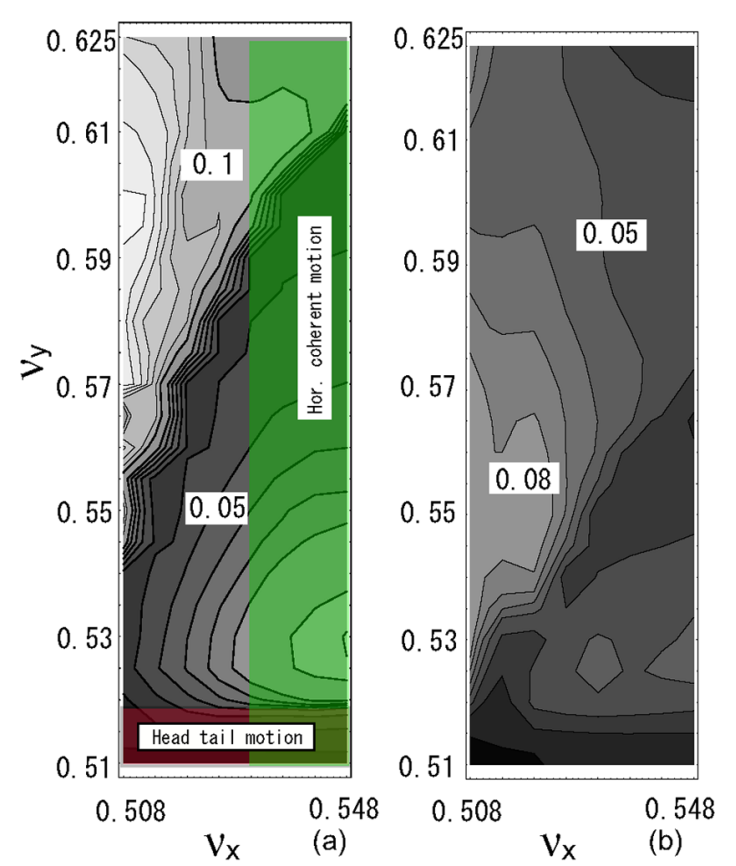

FIG. 6. (Color) Beam-beam parameter $\xi_{y}$ in $x-y$ tune space. The beam-beam parameter for the crossing angles of 0 and $11 \mathrm{mrad}$ are depicted in (a) and (b), respectively. The contour lines are drawn every 0.01 , and lighter gray corresponds to higher beam-beam parameter. 

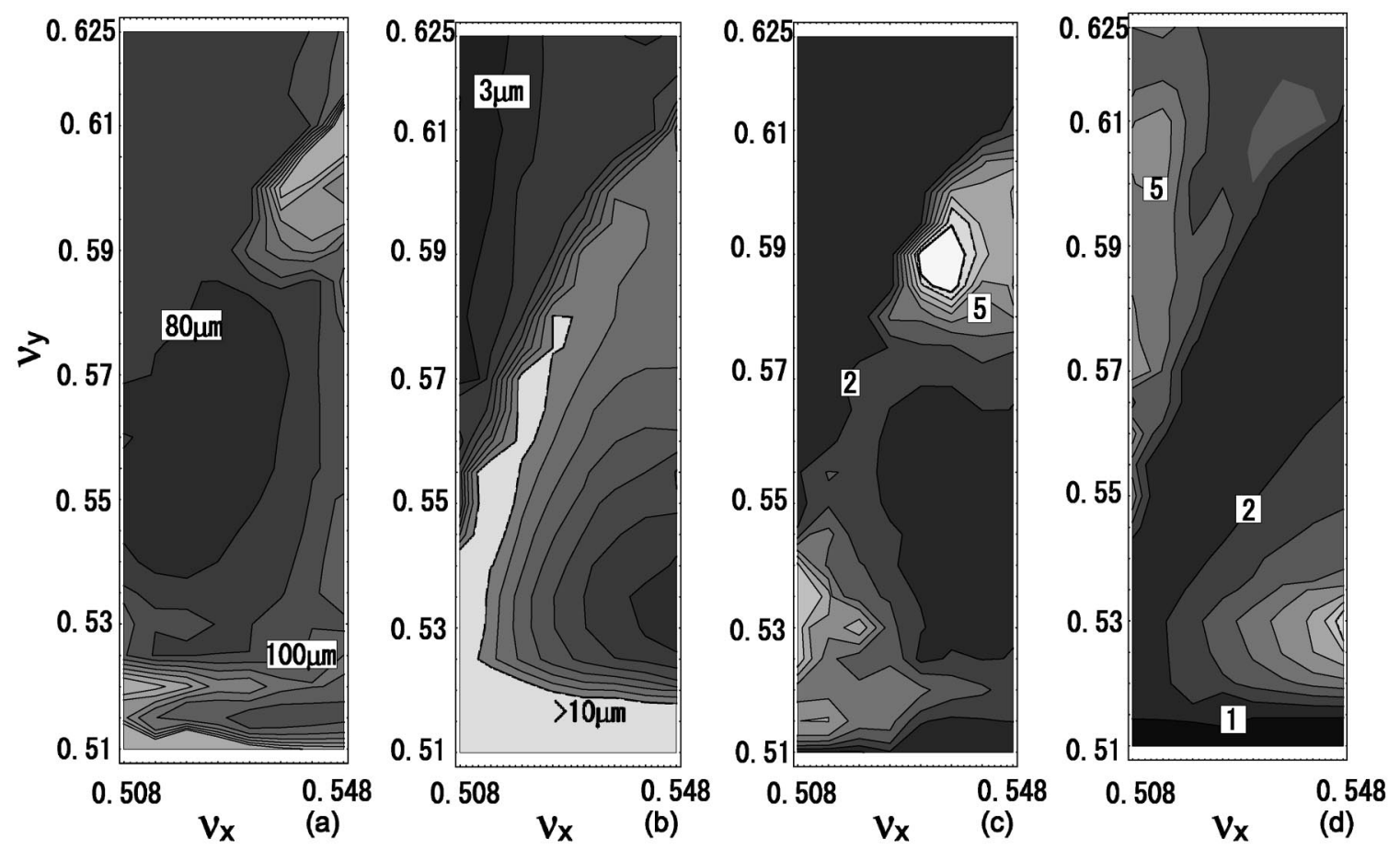

FIG. 7. Beam size and kurtosis in $x-y$ tune space for zero-crossing angle. The horizontal and vertical beam sizes are depicted in (a) and (b), respectively. The horizontal and vertical kurtosis are depicted in (c) and (d), respectively. The contour lines are drawn every $10 \mu \mathrm{m}, 1 \mu \mathrm{m}$, and 1 for horizontal, vertical beam size, and kurtosis, respectively, and lighter gray corresponds larger beam size and kurtosis.
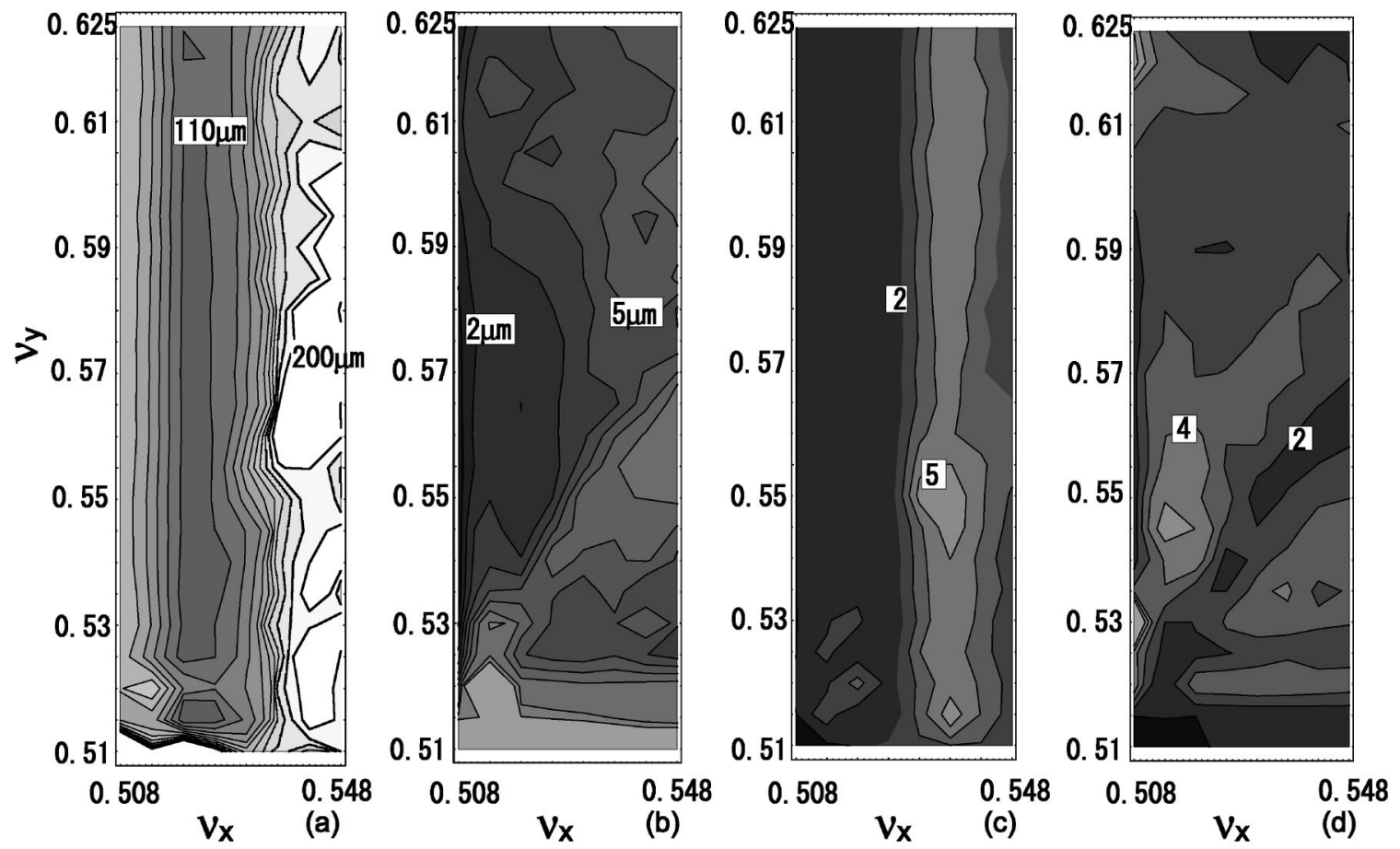

FIG. 8. Beam size and kurtosis in $x-y$ tune space for crossing angle of $11 \mathrm{mrad}$. The horizontal and vertical beam sizes are depicted in (a) and (b), respectively. The horizontal and vertical kurtosis are depicted in (c) and (d), respectively. The contour lines are drawn every $10 \mu \mathrm{m}, 1 \mu \mathrm{m}$, and 1 for horizontal, vertical beam size, and kurtosis, respectively, and lighter gray corresponds larger beam size and kurtosis. 
vertical size of Fig. 7(b). Smaller beam size is correlated with larger vertical kurtosis, as shown in Figs. 7(b) and $7(\mathrm{~d})$. The kurtosis, at the best operating point, $\left(\nu_{x}, \nu_{y}\right)=$ $(0.518,0.58-0.6)$, was $\left(k_{x}, k_{y}\right)=(1.5,5)$; i.e., the horizontal distribution is close to Gaussian but the vertical distribution is strongly distorted. The horizontal size did not have a correlation with the beam-beam parameter. The beam size and kurtosis have a positive correlation in horizontal: larger size is correlated to larger kurtosis as shown in Figs. 7(a) and 7(c).

The behavior of the beam-beam parameter in Fig. 6(b), which is given for a crossing angle of $11 \mathrm{mrad}$, is reflected in the vertical size of Fig. 8(b) again. The horizontal kurtosis is $1-1.5$ for $\nu_{x} \leq 0.53$, as shown in Fig. 8(c). The vertical kurtosis is high, 3-4, for the operating point with the highest beam-beam parameter as shown in Fig. 8(d). The horizontal size is minimum at $\nu_{x} \sim 0.52$. It does not seem to contribute to the beambeam parameter because the vertical size seems to behave in a way to cancel the gain due to the tunes. The kurtosis is $\left(k_{x}, k_{y}\right)=(1.5,1.5-2.5)$ for the operating point of $(0.508-0.518,0.58)$ shown in Fig. 3. In such a case, Gaussian approximation gives similar results to those obtained by the PIC model.

Coherent motions were sometimes seen at the tune survey. For higher horizontal tune, $\nu_{x}>0.55$, coherent motion limits the luminosity remarkably $(\xi \sim 0.01)$. No clear vertical coherent motion was seen in the tune area $\nu_{y} \geq 0.515$. For low vertical tune, $\nu_{y}=0.51$, vertical coherent motions in $\langle y\rangle$ and $\langle y z\rangle$, which may be due to the two-stream type of instability [21], were seen.

\section{E. Crab cavity}

So far, we have neglected the difference between true head-on collision without crossing angle and crab collision with a finite crossing angle. As discussed in Sec. II, crab cavities [24] create $z$ dispersion $\zeta_{x}$ at the collision point due to the transfer matrix $(6 \times 6)$ with a finite $(2,5)$ component. The dominant linear term in the transformation [Eq. (1)] caused by crossing angle was canceled by the $z$ dispersion. The nonlinear term of the transformation must be studied to confirm the validity of crab crossing.

Figure 9 shows the luminosity evolution for head-on collision without crossing angle and crab collision with a finite crossing angle $11 \mathrm{mrad}$. The two lines completely agree with each other; that is, the crab collision realizes the same performance as head-on collision without crossing angle.

Crab cavities control not only the dispersion $\zeta_{x}$ but also the derivative of the dispersion $\zeta_{x}^{\prime}=\Delta x^{\prime} / \Delta z$. The luminosity was evaluated as a function of $\zeta_{x}^{\prime}$. The reduction of luminosity or beam-beam parameter $\left[L\left(\zeta_{x}^{\prime}\right) / L(0)\right.$ or $\left.\xi_{y}\left(\zeta_{x}^{\prime}\right) / \xi_{y}(0)\right]$ was $98 \%$ and $93 \%$ for $\zeta_{x}^{\prime}=0.05$ and 0.1 , respectively. $\zeta_{x}^{\prime}$ can be reduced further due to the control of phase advance and other optics parameters between the

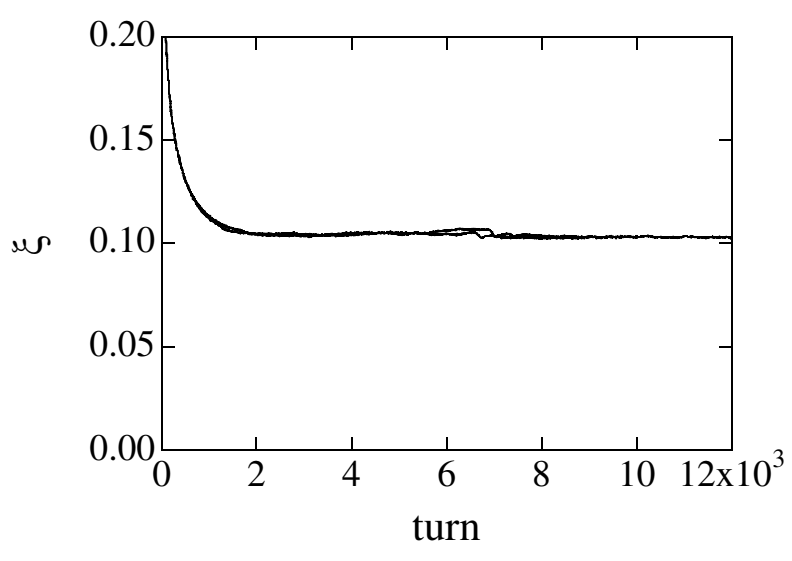

FIG. 9. Evolution of luminosity for head-on collision without crossing angle and crab collision with a finite crossing angle, $11 \mathrm{mrad}$.

crab cavity and the collision point [25]; therefore the effects of $\zeta_{x}^{\prime}$ do not need to be take into account.

\section{CONCLUSION}

We studied the beam-beam effect of collisions with or without crossing angle using weak-strong and strongstrong simulations.

Three-dimensional formalism, which is essential in order to study the effect of crossing angle, was developed. The method of synchrobeam mapping used for Gaussian approximation is extended for the PIC model; that is, the potential is interpolated along the longitudinal direction. This procedure reduces the number of longitudinal slices, with the result that computation time was reduced. The number of longitudinal slices required reduces to 5; otherwise 20-30 slices are needed to get the numerical convergence. This improvement allows us to carry out the strong-strong simulation for various parameters.

The beam-beam limit is caused by distortion from the Gaussian distribution for collision without crossing angle. The beam-beam limit was around $\xi \sim 0.1$ as is also discussed in Ref. [15]. The simulations using the Gaussian approximation were not able to reliably predict the beam-beam limit.

For collision with finite crossing angle of $11 \mathrm{mrad}$, the beam-beam parameter is limited to a lower value, around $\sim 0.06$ in both the weak-strong and strong-strong simulations. This fact suggests that the mechanism of the beambeam limit is different from that for the head-on collision.

We studied the diffusion caused by the beam-beam interaction. For zero-crossing angle, the diffusion was very weak for the Gaussian and the distorted beam [16]. The horizontal crossing angle induced both horizontal and vertical diffusion. The vertical diffusion is comparable with the radiation excitation, while the horizontal one is much smaller. The luminosity can be considered to be degraded by the diffusion. 
The diffusion due to the beam-beam interaction should be studied in more detail. Many studies have been done for a halo formation with a weak-strong model $[23,26,27]$. We propose diffusion as a mechanism that causes the beam-beam limit for collision with finite crossing angle. It is important to understand the beambeam limit in hadron colliders. If the beam-beam limit in proton colliders is determined by diffusion, it is essential to include errors, crossing angle, and longitudinal dimension in the simulations.

The tune survey was performed for the crossing angles of 0 and $11 \mathrm{mrad}$ using the strong-strong simulation. The tune operating point is essential for achieving a high beam-beam parameter. Especially, the best operating tune is different between head-on $\left(\nu_{x}, \nu_{y}\right)=(<0.51,0.58-0.6)$ and crossing collisions $(<0.51, \sim 0.55)$.

A crab cavity generates the $z$ dispersion $\zeta_{x}$ at the collision point. The $z$ dispersion, which is induced by the finite crossing angle in the one turn map, can be canceled by the $z$ dispersion due to the crab cavity. Small kinematic effects which remained in the one turn map did not affect the luminosity performance. Crab cavities, which realize the head-on collision effectively, can be expected to double the luminosity $(\xi \geq 0.1)$.

PEP-II has already been operated with the head-on collision. The beam-beam parameter is around 0.05 . Basically, PEP-II should benefit from the head-on collision scheme and be able to deliver higher luminosity. Simulation has shown that it is limited by $x-y$ coupling and the vertical dispersion in the machine in the present stage [28]. We believe that a fine-tuning of the optics parameters at the collision point and further reduction of the vertical dispersion in the arcs will improve the PEP-II performance.

\section{ACKNOWLEDGMENTS}

The authors thank members of the KEKB and PEP-II commissioning groups for fruitful discussions. This work was supported by the Japan-U.S. collaboration plan. They are grateful to the KEK computing center for supercomputer support. They also thank Thomas Knight for reading this manuscript. This work was partially supported by the Department of Energy under Contract No. DE-AC03$76 \mathrm{SF} 00515$.

[1] K. Oide and K. Yokoya, Phys. Rev. A 40, 315 (1989).

[2] K. Hirata, Phys. Rev. Lett. 74, 2228 (1995).

[3] KEKB Design Group, Technical Report No. KEK-report-95-7, 1995.

[4] M. Tawada, K. Ohmi, and Y. Funakoshi (to be published).

[5] Y.S. Derbenev and A. N. Skrinsky, in Proceedings of the 3rd All-Union Meeting on Charged Particle Accelerator, Moskow, 1972 (unpublished), p. 386.
[6] V.V. Danilov, P. M. Ivanov, E. A. Perevedentsev, E. A. Simonov, and A. N. Skrinsky, in Proceedings of the Workshop on Beam-Beam and Beam Radiation Interactions, Los Angeles, CA, 1991 (World Scientific, Hackensack, NJ, 1991), Vol. 1, p. 1.

[7] K. Hirata, H. Moshammer, and F. Ruggiero, Part. Accel. 40, 205 (1993).

[8] Y. Cai, A.W. Chao, S. I. Tzenov, and T. Tajima, Phys. Rev. ST Accel. Beams 4, 011001 (2001).

[9] E. B. Anderson and J.T. Rogers, in Proceedings of the Workshop on Beam-Beam Effects in Circular Colliders, Batavia, IL, 2001, edited by T. Sen and M. Xiao (FERMILAB-Conf-01/390-T), p. 136.

[10] M. Tawada et al., in Proceedings of the Workshop on Beam-Beam Effects in Circular Colliders, Batavia, IL, 2001 (Ref. [9]), p. 17.

[11] K. Ohmi, M. Tawada, and K. Oide, in Proceedings of the Particle Accelerator Conference (PAC03), Portland OR, 2003 (IEEE, Piscataway, NJ, 2003).

[12] K. Akai et al., Nucl. Instrum. Methods Phys. Res., Sect. A 499, 191 (2003).

[13] J. Augustin, Orsay Report No. 36-69, 1969.

[14] K. Ohmi, K. Hirata, and K. Oide, Phys. Rev. E 49, 751 (1994).

[15] K. Ohmi, M. Tawada, Y. Cai, S. Kamada, K. Oide, and J. Qiang, Phys. Rev. Lett. 92, 214801 (2004).

[16] K. Ohmi, M. Tawada, and K. Oide, KEK Report No. 2003-111, 2004.

[17] K. Ohmi, Phys. Rev. E 62, 7287 (2000).

[18] K. Ohmi and A. Chao, in Proceedings of the MiniWorkshop on Electron-Cloud Simulations for Proton and Positron Beams (ECLOUD'02), Geneve, 2002, edited by G. Rumolo and F. Zimmermann (CERN Report No. CERN-2002-001), pp. 227-234.

[19] M. Bassetti and G. Erskine, CERN Technical Report No. ISR TH/80-06, 1980.

[20] S.V. Milton and L.Z. Rivkin, in Proceedings of the European Particle Accelerator Conference (EPAC90), Nice, France, 1990, edited by G. Plass (Editions Fontieres, Yvette Cedex, France 1990), Vol. 1, pp. 255257.

[21] E. A. Perevedentsev and A. A. Valishev, Phys. Rev. ST Accel. Beams 4, 024403 (2001).

[22] K. Ohmi, M. Tawada, K. Oide, and S. Kamada, in Proceedings of the 3rd Asian Particle Accelerator Conference (APAC04), Gyeongju, Korea, 2004 (KEKReport No. 2004-13, 2004).

[23] J. L. Tennyson, in Physics of High Energy Particle Accelerators, edited by R. A. Carrigan, F. R. Huson, and M. Month, AIP Conf. Proc. No. 87 (AIP, New York, 1982), pp. 345-394.

[24] K. Akai and Y. Morita, KEK Report No. 2003-123, 2003.

[25] H. Koiso and A. Morita (private communication).

[26] T. Chen, J. Irwin, and R. Siemann, Phys. Rev. E 49, 2323 (1994).

[27] D. Shatilov, Part. Accel. 52, 65 (1996).

[28] Y. Cai, in PEP-II Machine Advisory Committee Meeting, 2003. 